\title{
The un-acknowledged relationship between shared parental leave, breastfeeding and workplace support in UK Universities
}

\author{
Vol: 2, Issue: 12 \\ December/2021 \\ DOI: http://dx.doi.org/10.47742/iibssr.v2n12p1 \\ https://ijbssrnet.com/index.php/ijbssr
}

\author{
Dr. Ernestine Gheyoh Ndzi \\ York Business School, \\ York St John University \\ Lord Mayor's Walk, York YO31 7EX \\ Email: e.gheyohndzi@yorksj.ac.uk \\ UK \\ Dr. Anjali Raj Westwood \\ York Business School \\ York St John University \\ Lord Mayor's Walk, York YO31 7EX \\ Email: a.raj@yorksj.ac.uk \\ UK
}

\section{A R T I C L E I N F O \\ Article history: \\ Received: $\quad$ Dec 2021 \\ Revised: $\quad$ Dec 21,2021 \\ Accepted: $\quad$ Dec 27, 2021 \\ Publication: Dec 31, 2021 \\ DOI: $10.47742 /$ ijbssr.v2n12p1}

https://creativecommons.org/licenses/by/4.0/

\begin{abstract}
A B S T R A C T
Gender equality is integral to a universities' strategy and agenda. However, there is a gap when looking through the lens of shared parental leave (SPL) or breastfeeding. This research investigates the causal relationship between SPL, breastfeeding, and workplace support. A survey targeting women working in UK Higher Education Institutions was conducted between February and July 2021, and 49 completed responses were recorded. Women reported knowledge of SPL but not all workplaces were offering SPL, it wasn't easily accessible and often too complex to understand. Breastfeeding policies and workplace resources were minimal. Lack of breastfeeding support on return to work affected women's decision to take SPL. Recommendations include the creation of more accessible policies (SPL and breastfeeding), providing examples of parents who have utilized SPL, dissemination of information on SPL and breastfeeding when women announce their pregnancy and on return to work, and adequate resources in the workplace to support breastfeeding mothers.

Keywords: Shared Parental Leave, Breastfeeding, Maternity Policies, Organisational Culture, Education
\end{abstract}

\section{Introduction}

Higher Education Institutions are classed under high skilled sectors and the question to be addressed is how women in academia are supported by their institutions on shared parental leave and breastfeeding upon return to work. While many Higher Education institutions have gender equality on their strategic plan and many are applying for the Athena Swan accreditation, there doesn't seem to be much in the way of uptake of SPL or visible breastfeeding policies and signage in most institutions. Women of childbearing age are an integral part of the workforce at any given time. The growth of women entering the labor force has been steadily increasing in the UK with about $72 \%$ of women aged 1664 employed by June 2020 (ONS, 2021). Without this workforce, organizations will struggle to successfully and sustainably function, and they contribute to the national economies. Women may choose to start a family whilst they are employed and return to work after the birth of their baby. Though the importance of maternity leave has been acknowledged widely in all universities, shared parental leave (SPL) and breastfeeding upon return to work has not seen much encouragement. The UK Coalition Government, in 2013, introduced the policy on SPL as a key initiative to increase gender equality in the workplace and drive culture change. The policy allows mothers to share their maternity leave with their partners which means mothers could return to work much earlier after birth and dads could take longer leave to bond with the child. This research explains how SPL and breastfeeding practices work in UK Higher Education Institutions. It highlights the relationship between SPL, breastfeeding, workplace culture, and support. The research also makes recommendations for policy changes to encourage workplace support which could boost SPL take-up and breastfeeding practices.

\section{Background}

Shared parental leave represents a significant milestone in policy change towards achieving gender equality in the workplace and encouraging fathers to spend more time with their families. The policy has the potential to change the perception of men as breadwinners and women as caregivers. Existing literature on SPL has not explored the relationship and impact of SPL on breastfeeding and vice versa. Research has demonstrated that SPL improves both parents' sense of trust and confidence in their parental skills (Haas \& Hwang, 2008), fathers engaging more in childcare tasks and deriving more satisfaction engaging with their children (Almqvist \& Duvander, 2014), reducing parenting stress (Lidbeck et al.,2018) and enhances parents' relationship (Duvander et al., 2017). Plantin et al. (2011) argued that a father's involvement in childcare has a positive impact on the child's social, behavior, and psychological development and wellbeing. 


\title{
International Journal of Business and Social Science Research
}

\author{
Vol: 2, Issue: 12 \\ December $/ 2021$ \\ DOI: http://dx.doi.org/10.47742/ijbssr.v2n12p1 \\ https://ijbssrnet.com/index.php/ijbssr
}

However, uptake of SPL remained low since its introduction in 2015 (Birkett and Forbes, 2019). The challenges of SPL include lack of awareness of the policy and its practicality (Ndzi, 2017), low pay and lack of mother's desire to share her maternity leave (Twamley and Schober, 2019), the complexity of the policy, and poor communication at work (Birkett and Forbes, 2019); and lack of support for fathers in the workplace (Brandth and Kvande, 2019)

The benefits of breastfeeding for the mother and infants are widely known. The world health organization recommends six months of exclusive breastfeeding and two years of complementary feeding of breastmilk and solids for optimal child development and nutrition (WHO,2002). Working mothers should not have to choose between breastfeeding their babies and getting back to work. The benefit of breastfeeding extends to employers because it reduces work, absenteeism, and increases employee morale and retention (Cohen \& Mrtek, 1994; Cohen et al., 1995). In addition to the benefits, breastfeeding can provide to the employer, it has been found to have many health benefits such as reducing the risk of diarrhea, allergies, leukemia, infant sudden death, lymphoma, for the child (Goldman, 2000). Advantages to the mother include reduced risk of breast cancer and rates of osteoporosis (Boswell-Penc and Boyer, 2007), reduce the potential for type 2 diabetes and obesity (Victora et. al., 2016), reduce risk of asthma (Lodge et al., 2015) and other conditions such as morbidity (Sankar et. al., 2015). Research demonstrates that working mothers do not tend to breastfeed for long periods (Hawkins et al.,2007) and are more likely to give up breastfeeding upon return to work (Desmond and Meaney, 2016). Lack of support for breastfeeding women returning to work is a key challenge to breastfeeding rates (Gatrell, 2007) and the idea behind SPL is for mothers to return to work early after childbirth. SPL then poses a challenge on breastfeeding for women returning to work as well as breastfeeding poses a challenge on SPL for mothers who intend to stay off work longer for breastfeeding purposes.

In the UK, there is no national policy on breastfeeding in the workplace. Women have a statutory right to paid rest breaks whilst breastfeeding. Though the statutory right covers health and safety, flexible working hours, protection from indirect sex discrimination, rest, and protection from harassment, there is no further detail on the duration of breaks, or other breastfeeding/expressing related facilities (Maternity Action, 2021). Facilities like clean and private rooms, secure and clean fridges, a sufficient number of breaks, etc are suggestions but not mandatory (NHS, 2021). This leaves space for subjective interpretation of the situation leading to a range of breastfeeding policies from very supportive to a total lack of support.

The uptake of SPL has been low, and breastfeeding could be one of the causal factors for this which makes it imperative to study the effect and relationships of SPL on breastfeeding and vice versa. This paper is a part of a larger study investigating the existing policies within the Higher Education Institutions in the UK.

\section{Methods}

This article identifies a key issue that has been missed in existing articles which is the relationship between SPL, breastfeeding, and workplace support. An online survey was used to collect data. The survey questionnaire was designed by the researchers. The questionnaire had three key parts to it: demographics shared parental leave and breastfeeding. The questions were designed to understand participants' understanding and experience of workplace practice about SPL and breastfeeding and how that might have influenced their decisionmaking. The time required to complete the questionnaire was 1520 mins which a significant part of the questionnaire being intext discussion on the participant's experience. The intext content was used to analyze the data. The survey received 73 responses of which 24 were excluded from the analysis because they were incomplete. Whilst 49 responses were analyzed, we consider this robust because the questionnaires were heavily qualitative than quantitative.

Convenience sampling was the chosen method to collect responses (Bryman \& Bell, 2011) Women who had given birth on or after the 5th of April 2015 (which is when SPL became available) and are employed in Higher Education Institutions in the UK were invited to participate. The survey link was shared via social media accounts such as Facebook, LinkedIn, Twitter, and Instagram.

Ethical approval was granted by the school ethics committee of the University on the 24th of February 2021. Descriptive analysis was used to analyze the qualitative data (Bryman \& Bell, 2011). All data was anonymized to preserve confidentiality.

\section{Results}

A total of seventy-three responses were received. Twenty-four responses were excluded as they were incomplete. A total of forty-nine responses were considered for data analysis. As the data set was small, it was analyzed using MS Excel.

\section{Demographics}

Participants ranged from 21 to $45+$ years of age. The maximum representation $(n=21)$ was in the age group $36-40.63 \%$ of all respondents held a Ph.D., $16 \%$ held a Masters's, $16 \%$ a first degree and $4 \%$ were at O/A Levels. $78 \%$ of respondents were of British/Welsh/Scottish/Irish heritage, $2 \%$ each of Asian/Asian British or Black/African/Caribbean/Black British, and 18\% belonged to none of the above options. $90 \%$ already had a child/children and $10 \%$ were pregnant while completing the survey. $57 \%$ had one child, $35 \%$ had two and $6 \%$ had three children. Only $2 \%$ were currently pregnant with their first child.

$51 \%$ of respondents were in full-time employment, $45 \%$ part-time and $4 \%$ were on other contracts. The majority of the respondents were at an entry-level (59\%) or non-managerial role and only one respondent was at an executive level. Combined family income was a key demographic to this study. The most common (22\%) earned $£ 71,000$ to $£ 80,000$ per annum. $6 \%$ had an income over $£ 100,000$ per annum and $4 \%$ reported the lowest end of the scale of below $£ 20,000$ per annum. A majority $(67 \%)$ of the respondents reported earning between $£ 41,000$ - $£ 80,000$ per annum. 


\title{
International Journal of Business and Social Science Research
}

\author{
Vol: 2, Issue: 12 \\ December $/ 2021$ \\ DOI: http://dx.doi.org/10.47742/ijbssr.v2n12p1 \\ https://ijbssrnet.com/index.php/ijbssr
}

SPL

It was necessary to ensure that the data included those that had taken SPL and those that had not. 54\% of the respondents had taken SPL. When asked about knowledge and source of information on SPL, $51 \%$ of respondents reported that they knew what SPL was, $45 \%$ had minimal knowledge of it, $2 \%$ had never heard about it and $2 \%$ had heard it but didn't know what it was. Looking into where they first heard about SPL, the most common answer was Employer (24\%) followed by Government website $(22 \%)$. This feeds into their awareness of workplace policy on SPL. $71 \%$ of the respondents were aware of the existence of SPL policy in their workplace. $24 \%$ did not know if such a policy existed, $2 \%$ did not have it at their workplace and $2 \%$ never heard of it (the same $2 \%$ from the first question on knowledge of SPL as a policy).

In terms of access to SPL information and understanding how the policy works in practice, 32 participants knew where to find their workplace's SPL policy, 4 did not know; 15 said that their SPL policy is difficult to read and understand whereas 11 said it was easy to read and understand; 7 said their employers did not understand SPL and couldn't explain it to them whereas 7 others said their employer was well informed and provided them with useful explanations which aided their decision making; 6 said their workplace did not enhance SPL pay and 7 said their workplace did enhance SPL pay. Only 4 were given other examples of colleagues who took SPL.

When asked about how their employer supported SPL, four participants reported no support or mention of SPL by HR.

Most participants reported that they were given the required SPL forms to fill out of they wanted to take SPL. However, some of the respondents reported having very helpful discussions on what SPL is and how it could apply to them, whilst others reported not having any such discussion or very basic discussion that did not help much with their understanding of SPL as explained by one of the mothers:

"It took me weeks to get confirmation from HR o how they would pay me. I keep querying this way of organizing pay and had to chase up again and again, and they'd take weeks to get back to me. If I'd known they were going to dock my full pay weeks I could have gone on maternity leave earlier and had all six weeks of full pay, but by the time they confirmed it I was already on leave."

A key part of this survey was identifying what the participants needed from their employer to increase uptake of SPL. Three key themes arose include Accessible information, financial constraints, and breastfeeding.

Participants highlighted the need for accessible and easy to understand information about SPL and its implications: benefits'

"More knowledge of SPL and implications e.g tax and

"Employer taking time to educate you about rights and policies", "A clear understanding of how it could be taken and any implications."

Support from the partner's employer and the pay gap between the participant and her partner was a big reason for many participants to not take SPL:
"It seems very complicated, with barriers between my employer and my partner's (non-academic) employer", "I thought my husband's employer would need to support him taking SPL, which it did not", "Difficult as I am the lower earner and there is not much to change that?".

Out of the 13 participants who took SPL, five chose to return after 6 months because it would affect breastfeeding, six would have to/have expressed at work and two felt taking SPL and returning to work early did not affect breastfeeding. More on breastfeeding is presented in the next section.

\section{Breastfeeding}

All participants indicated that they were aware of the suggested benefits of breastfeeding. All except one said that the health benefits determined their decision to breastfeed their baby. $90 \%$ of respondents breastfed/plan to breastfeed their children and $10 \%$ planned to mixed feed. Going further into their plans for breastfeeding, $51 \%$ planned to breastfeed for over 13 months, $24 \%$ for $10-12$ months, $12 \%$ for $7-9$ months, $10 \%$ for $4-6$ months, and only $2 \%$ for under 3 months. Matching that to how long respondents with children $(n=44)$ breastfed for, only $66 \%$ breastfed for over 13 months, and 5\% each for 4-6 months, 7-9 months, and 10-12 months. When looking into plans for exclusive breastfeeding, 82\% planned to exclusively breastfeed for 6 months. Six months is the recommended duration by WHO. The rest were sparsely spread across the 6 months or were unsure. Matching that to how long women $(n=44)$ exclusively breastfed, $77 \%$ exclusively breastfed for six months and $14 \%$ only for one month. The rest were spread between 1 and 6 months.

Unlike the high numbers of participants aware of SPL policies, when asked about breastfeeding policies, only $16 \%$ of participants had encountered a workplace policy on breastfeeding. $7 \%$ said there was no policy and 53\% were not aware of such a policy existed. We presented the participants with a series of statements regarding when they got to know about breastfeeding policies at their workplace, asking them to choose all that apply. 8 of them had breastfeeding policies included in their maternity policy, 3 asked colleagues when they were pregnant, 1 asked her employer when she was pregnant; when they were ready to return to work, 12 asked their employer and 7 asked colleagues, 3 were given the information by their employer; 4 saw breastfeeding signages at work and 10 said they had no breastfeeding support at all; 1 was told about it during induction, and 14 did not choose any.

$41 \%$ of participants indicated that they returned/will return to work between $25-39$ weeks after childbirth and $47 \%$ after 40 weeks. Only $10 \%$ indicated that they would return before 24 weeks. This could be linked to how it would affect exclusive breastfeeding. When asked about this, 29\% said that if they returned to work before 6 months, it would affect exclusive breastfeeding. 18\% said it wouldn't affect and 37\% were unsure if it would. $16 \%$ chose not to answer this question.

This led to exploring if the participants were comfortable expressing milk at work. 35\% said they would be comfortable with it, $49 \%$ said they wouldn't be comfortable but will do if they need to, $6 \%$ will not express breastmilk at work and $10 \%$ were unsure of it. Breastfeeding facilities influence women's comfort 


\title{
International Journal of Business and Social Science Research
}

\author{
Vol: 2, Issue: 12 \\ December/2021 \\ DOI: http://dx.doi.org/10.47742/ijbssr.v2n12p1 \\ https://ijbssrnet.com/index.php/ijbssr
}

levels. $73 \%$ reported that breastfeeding facilities were not signposted at their workplace. Only $10 \%$ responded Yes to clear signposting of facilities. $16 \%$ were not aware of it at all.

When asked about the type of facilities available, $32 \%$ did not know what facilities were available, $59 \%$ were given adequate breaks to express breastmilk. However, only $41 \%$ had access to private rooms with sockets, $27 \%$ had additional access to a fridge and $12 \%$ had a nursery onsite along with breaks, private rooms, sockets, and a fridge. $8 \%$ of the respondents had no facilities at all. $32 \%$ did not know what facilities were present at work.

When asked to detail how their workplace support affects their decision to breastfeed, most of the participants were determined to breastfeed irrespective of workplace support. Some statements given were,

"It didn't. I was planning to breastfeed regardless of my employer's policies",

"My workplace was not a factor in my decision to breastfeed. It was based upon what was best for my child and myself.".

Four of the participants decided to take a full year of maternity leave due to the unsupportive breastfeeding policies and the lack of adequate infrastructure. The participants highlighted that the lack of support makes the experience more difficult than it should be.

"Breastfeeding facilities were not adequate. Had to use porters lodge (small room under stairs) and ask male porters at reception every time I wanted to express so not fully private. I had to negotiate to express around their lunch breaks and they would make comments around expressing like 'it's my turn next. Felt like I had to do this on my lunch break and I feel it did shorten the amount of time I ended up breastfeeding for as too complicated to fit around work/not comfortable or private environment "

Two participants had their own private offices which played a key role in their comfort levels of expressing milk at work. In larger campuses, distance to the breastfeeding rooms are a critical measurement that is often forgotten as detailed by this participant:

"It helps to know it is available, however, whilst facilities are available in some buildings on campus, the nearest breastfeeding room was a 10 min walk away, and when I first returned, I need to express 3-4 times a day. Because of this, I used the shower room in my building instead (no windows, lockable door, but no other facilities described above) ".

The lockdowns due to the pandemic were helpful for some of the participants who found it comfortable to breastfeed their children while working from home.

When investigated if the return to work will affect breastfeeding, $61 \%$ of respondents said that they would have to express at work. Only $29 \%$ said it wouldn't affect breastfeeding. $8 \%$ said they would have to stop breastfeeding and $2 \%$ were unsure.

Regarding the link between taking SPL and breastfeeding, 2\% said they would have to stop breastfeeding, $37 \%$ would have to express at work, $22 \%$ wouldn't take SPL as they wanted their entire maternity leave, 24\% wouldn't return before six months, 10\% said it wouldn't make a difference and $2 \%$ were unsure. The decision to take SPL did not significantly affect the duration of breastfeeding amongst the participants.

\section{Discussion}

The results of this survey have highlighted challenges faced by women within the HEI sector in the UK transitioning into motherhood and retaining to work. The survey was set out to understand the relationship between SPL, breastfeeding, and workplace support. This is because SPL is a new policy that has the potential to change cultural norms where men are regarded as breadwinners and women as primary caregivers. Shared parental leave gives the parents the chance to share the mother's maternity leave. This means that the mother could go back to work much earlier after childbirth and the father could take longer than two weeks of leave to spend and bond with the baby. While most of the participants acknowledged that there was an SPL policy in their workplace, many did not hear about it from their employers. This is a challenge to the uptake of SPL if parents don't know about the policy to be able to make an informed decision which is a point Ndzi (2017) made that for SPL to be effective, awareness of its existence is crucial. Furthermore, some of the participants reported that their workplaces had no policy at all on SPL. The lack of SPL policy in the workplace could be interpreted by staff as non-support. This could deter staff from asking about the policy even if they know from others that such a policy exists. The support of the workplace is crucial for staff awareness and takeup of SPL. Although 54\% of the respondents had taken SPL, the data suggest that the experience differed with some having great support and others much less so.

Policy accessibility in terms of where to find it and how easy it is for staff to understand what it means and how it could apply to them was one of the key challenges identified. Participants reported that although they knew about SPL and their employers had a policy on SPL, they found it difficult to locate the policy on the employer's system. Others reported that whilst they could find the policy, they were unable to understand how it will apply to them in practice. SPL policy has been criticized for its complexity (Birkett and Forbes, 2019) and employers, therefore, need to ensure that they bring the policy to the attention of the staff, explain the practicality of the policy for the staff to be able to make an informed decision on it. Some of the participants reported that their human resources personnel were unable to explain the SPL policy to them which could result in a high level of distrust in the human resources systems and support for the staff.

Some of the participants discussed how helpful it was that they had access to examples of colleagues that had taken SPL. These examples made it much easier for the staff to understand what SPL meant and how it could work for them. It also signaled to the colleagues that their employer was happy and supportive of them taking SPL. This is a point that links to a comment made by one of the mothers about the lack of support from her partner's (father) employer. As mentioned earlier, the rationale for SPL is to encourage gender equality, but if employers are not supporting fathers to take the leave and employers are not supporting mothers 


\title{
International Journal of Business and Social Science Research
}

\author{
Vol: 2, Issue: 12 \\ December $/ 2021$ \\ DOI: http://dx.doi.org/10.47742/ijbssr.v2n12p1 \\ https://ijbssrnet.com/index.php/ijbssr
}

to take the leave, SPL takes up will remain low. This low uptake will denote continuity in gender discrimination and the gender pay gap.

Against the backdrop of SPL, was the impact of breastfeeding mothers returning to work after childbirth with the desire to continue breastfeeding. Mothers in our study were conscious about the benefits of breastfeeding and planned to breastfeed for a significant amount of time but it did not match the duration they did breastfeed for. The benefits of breastfeeding for the mother (Goldman, 2000; Boswell-Penc and Boyer, 2007), baby (Victora et. al., 2016; Lodge et al., 2015; Sankar et. al., 2015), and the organization (Cohen \& Mrtek, 1994) where the mother works, are widely known. It needs to be a norm for organizations to create an environment where their employees can flourish. WHO recommends 6 months of exclusive breastfeeding followed by complementary feeding up to 2 years (WHO,2002). It was evident from our research that a significant number of women did not want to return to work before the child was 25 weeks, as they were convinced that returning to work would affect exclusive breastfeeding. When they did return to work, it has its trickle effect on complementary feeding. The women are being put in an uncomfortable position where they must choose between their career and the baby. Either option will leave the mother feeling guilty.

An ideal infrastructural breastfeeding provision will include rest breaks, an accessible private room with sockets, a fridge for storage of milk, and the onsite nursery. Only $12 \%$ of the participants had these facilities. Providing the legal minimum of rest breaks in itself is not conducive for mothers to continue breastfeeding their babies. Mothers reported feeling frustrated having to use communal offices, shower rooms, or porter's lodges to express milk. A clean, private room is the minimum a breastfeeding mother returning to work requires.

When discussing a return to work, logistics of breastfeeding support aside, there is a physiological side to breastfeeding that is often not accounted for. Breastfeeding mothers need to express milk every few hours or else they have painful and swollen breasts, increased risk of infection, and back pain (Torgus \& Gotsch, 2004). Their milk supply will drop as the body uses the removal of milk as an indicator of how much to make in the first place. All the above causes significant physical and emotional strain on the mother. When women return to work after maternity, they may already feel disconnected, exhausted, and as if they are playing catch-up with their careers. The physical and emotional challenges around breastfeeding at work are an unfortunate and unnecessary addition to the list.

Workplace culture plays a key role when we look at breastfeeding at the workplace. Lack of a breastfeeding policy, signages, or discussions around this indicates that the organization is not openly advocating or supporting mothers to breastfeed or express when they return to work. Creating and implementing breastfeeding policies can support organizations to achieve gender equality, reduce the motherhood penalty and be an attractive place to work, which in turn will help with the attraction and retention of high-quality staff.

The dissemination of information on SPL and breastfeeding policies is another challenge. Women do not have one single point where they can gain all the information they need. It is spread across various sources- digital and interpersonal. In an ideal scenario, women should not have to go looking for it, it should be presented to them when they inform their manager that they are pregnant and when they return to work.

SPL has the potential to help mothers with their career progression, and for fathers to bond with their babies. To achieve higher uptake of SPL, employers need to proactively promote SPL by ensuring that the policy is clear and easy to understand with examples. The policy should be brought to the attention of any parent who notifies the employer of their intention to go on maternity, paternity, or adoption leave. Where employers are enhancing maternity and paternity leave, to enhance SPL pay. Line managers should help signpost staff to the available policies and discuss any support staff might need in deciding or taking SPL.

With regards to breastfeeding at work, the authors recommend that HEI's should design and develop breastfeeding policies that will apply to all employees (academic or professional services) after discussion with stakeholders. The policies should be matched with infrastructural changes such as a combination of dedicated breastfeeding rooms and private rooms which can be used for breastfeeding or expressing milk. The rooms should be easily accessible, and employees should have access to dedicated fridges to store the expressed milk. The rooms must be signposted on physical and digital campus maps for students and staff alike. Line managers and HR need to be sensitized towards the needs of women returning to work after having a baby. All the information regarding breastfeeding support and facilities should be given to women when they first approach with the news of their pregnancy and when they announce their return to work. A peer support group or a buddy scheme will also be a welcome addition.

\section{Conclusion}

One cannot achieve true gender equality without considering the effect of motherhood on a woman's career. There is a causal relationship between uptake of SPL and breastfeeding policies. Though the SPL policy was introduced to support gender equality in the workplace for parents, the lack of workplace support in explaining the complexity of the policy, enhancing the pay, and the lack of breastfeeding support for mothers returning to work, are key reasons for low uptake of SPL. The study established the relationship between SPL, breastfeeding, and workplace support and how it impacts the uptake of SPL. One limitation of this survey was the number of participants. However the data is suggestive of definite challenges faced by working women in HEI. This data has been further triangulated with policy analysis and in-depth interviews which are being written as separate papers. Recommendations for improving uptake of SPL and for creating a supportive breastfeeding environment have been provided. 


\title{
International Journal of Business and Social Science Research
}

\author{
Vol: 2, Issue: 12 \\ December/2021 \\ DOI: http://dx.doi.org/10.47742/ijbssr.v2n12p1 \\ https://ijbssrnet.com/index.php/ijbssr
}

\section{References}

Almqvist, A., Duvander, A. (2014). Changes in gender equality? Swedish fathers' parental leave, division of childcare and housework. Journal of Family Studies, 20(1), 19-27.

Birkett, H. and Forbes, S. (2019). Where's dad? Exploring the low take-up of inclusive parenting policies in the UK. Policy Studies, 40, 205-224.

Boswell- Penc, M. and Boyer, K. (2007) 'Expressing anxiety? Breast pumps usage in American Wage workplaces', Gender, Place and Culture, 14(5): 151-167.

Brandth, B. and Kvande, E. (2019). Workplace support of fathers' parental leave use in Norway. Community, Work \& Family, 22, 43-57.

Bryman, A., \& Bell, E. (2011). Business research methods. Oxford Univ. Press.

Cohen, R. J., Haddix, K., Hurtado, E., \& Dewey, K. G. (1995). Maternal activity budgets: Feasibility of exclusive breastfeeding for six months among urban women in Honduras. Social science \& medicine, 41(4), 527-536.

Cohen, R., \& Mrtek, M. (1994). The Impact of Two Corporate Lactation Programs on the Incidence and Duration of Breast-Feeding by Employed Mothers. American Journal Of Health Promotion, 8(6), 436-441. https://doi.org/10.4278/0890-1171-8.6.436

Cohen, R., Mrtek, M. B., \& Mrtek, R. G. (1995). Comparison of Maternal Absenteeism and Infant Illness Rates among Breast-Feeding and Formula-Feeding Women in Two Corporations. American Journal of Health Promotion, 10(2), 148-153.

Cohen, R., Mrtek, M., \& Mrtek, R. (1995). Comparison of Maternal Absenteeism and Infant Illness Rates among Breast-Feeding and Formula-Feeding Women in Two Corporations. American Journal Of Health Promotion, 10(2), 148-153. https://doi.org/10.4278/0890-1171-10.2.148

Desmond, D., Meaney, S. (2016) 'A qualitative study investigating the barriers to returning to work for breastfeeding mothers in Ireland', Int Breastfeed J, 11:16.

Duvander, A. Z., Haas, L., Thalberg, S. (2017). Fathers on leave alone in Sweden: Toward more equal parenthood? In O’Brien, M., Wall, K. (Eds.), Comparative perspectives on work-life balance and gender equality (pp. 125-145).

Gatrell, C. (2007) 'Secret and Lies: Breastfeeding and professional paid work', Social Science and Medicine, 65(2): 393-404

Goldman, A.S. (2000) 'Modulation of the gastrointestinal track of infants by human milk, interfaces and interactions: an evolutionary perspective', Journal of Nutrition, 130: 426-431.

Haas, L., Hwang, P. (2008). The impact of taking parental leave on father's participation in childcare and relationships with children: Lessons from Sweden. Community, Work \& Family, 11(1), 85-104.

Hawkins, S.S., Griffiths, L.J., Dezateux, C., Law, C. (2007) 'Millennium Cohort Study Child Health Group. Maternal employment and breast-feeding initiation: findings from the Millennium Cohort Study', Paediatr Perinat Epidemiol, 21(3): 242-7.

Lidbeck, M., Bernhardsson, S., Tjus, T. (2018). Division of parental leave and perceived parenting stress among mothers and fathers. Journal of Reproductive and Infant Psychology, 36(4), 406-420.

Lodge, C., Tan, D.J., Lau, M.X.Z., Dai, X., Tham, R., Lowe, A.J., Bowatte, G., Allen, K.J. and Dharmage, S.C. (2015) 'Breastfeeding and asthma and allergies: A systematic review and meta-analysis', Acta Paediatrica, 104: 38-53.

Maternity Action. (2021). Continuing to breastfeed when you return to work - Maternity Action. Maternity Action.

Retrieved 15 December 2021, from https://maternityaction.org.uk/advice/continuing-to-breastfeed-when-you-return-towork/.

Ndzi, E. (2017). Shared parental leave: awareness is key. International Journal of Law and Management, 59(6) 1331-1336.

NHS. (2021). Breastfeeding and going back to work. NHS. Retrieved 15 December 2021, from

https://www.nhs.uk/conditions/baby/breastfeeding-and-bottle-feeding/breastfeeding-and-lifestyle/back-to-work/.

Nowell S., Norris M., White E., and Moules J., (2017). Thematic Analysis: Striving to Meet the Trustworthiness Criteria: International Journal of Qualitative Methods, 16, pp. 1-13.

Office of National Statistics, 'Female employment rate (aged 16 to 64, seasonally adjusted)' (2021)

https://www.ons.gov.uk/employmentandlabourmarket/peopleinwork/employmentandemployeetypes/timeseries/lf25/lms accessed 30th June 2021.

Plantin, L., Olukoya, A. A., Ny, P. (2011). Positive health outcomes of fathers' involvement in pregnancy and childbirth paternal support: A scope study literature review. Fathering: A Journal of Theory, Research, and Practice about Men as Fathers, 9(1), 87-102.

Torgus, J., \& Gotsch, G. (2004). The womanly art of breastfeeding. La Leche League International. 


\section{International Journal of Business and Social Science Research}

Vol: 2, Issue: 12

December/2021

DOI: http://dx.doi.org/10.47742/ijbssr.v2n12p1

https://ijbssrnet.com/index.php/ijbssr

Twamley, K. and Schober, P. (2019). Shared Parental Leave: Exploring variations in attitudes, eligibility, knowledge and take up intentions of expectant mothers in London. Journal of Social Policy, 48(2), 387-407.

Victora, C., Bahl, R., Barros, A., França, G., Horton, S., Krasevec, J., Murch, S., Sankar, M., Walker, N., Rollins, N. (2016) 'Breastfeeding in the 21st century: Epidemiology, mechanisms', The Lancet, 387(10017): 475-490.

WHO. (2002). Infant and Young Child Nutrition. WHO. Retrieved from

https://apps.who.int/gb/archive/pdf files/WHA55/ea5515.pdf 